\title{
Neutrophil-epithelial crosstalk at the intestinal lumenal surface mediated by reciprocal secretion of adenosine and IL-6
}

\author{
Shanthi V. Sitaraman, ${ }^{1,2}$ Didier Merlin, ${ }^{1}$ Lixin Wang, ${ }^{1}$ Michelle Wong, ${ }^{1}$ \\ Andrew T. Gewirtz, ${ }^{1}$ Mustapha Si-Tahar, ${ }^{1}$ and James L. Madara ${ }^{1}$ \\ ${ }^{1}$ Epithelial Pathobiology Unit, Department of Pathology, and \\ ${ }^{2}$ Division of Digestive Diseases, Department of Medicine, Emory University, Atlanta, Georgia, USA
}

Address correspondence to: Shanthi V. Sitaraman, 1639 Pierce Drive, Room 2101 WMRB, Atlanta, Georgia 30322, USA. Phone: (404) 727-2430; Fax: (404) 727-5767; E-mail: ssitar2@emory.edu.

Mustapha Si-Tahar's present address is: Unité de Pharmacologie Cellulaire,

Unité Associée/Institut National de la Santé et de la Recherche Médicale 285, Institut Pasteur, Paris, France.

Received for publication November 14, 2000, and accepted in revised form February 7, 2001.

Adenosine is formed in the intestinal lumen during active inflammation from neutrophil-derived 5 ' AMP. Using intestinal epithelial cell line T84, we studied the effect of adenosine on the secretion of IL-6, a proinflammatory cytokine involved in neutrophil degranulation and lymphocyte differentiation. Stimulation of T84 monolayers with either apical or basolateral adenosine induces A2b receptor-mediated increase in IL-6 secretion, which is polarized to the apical (luminal) compartment. In addition, Salmonella typhimurium, TNF- $\alpha$, and forskolin, known inducers of IL- 6 secretion in intestinal epithelial cells, also stimulate IL- 6 secretion into the apical compartment. We show that IL6 promoter induction by adenosine occurs through cAMP-mediated activation of nuclear cAMPresponsive element-binding protein (CREB). We also show that IL-6 released in the luminal (apical) compartment achieves a sufficient concentration to activate neutrophils (from which the adenosine signal originates), since such IL- 6 is found to induce an intracellular $\left[\mathrm{Ca}^{++}\right]$flux in neutrophils. We conclude that adenosine released in the intestinal lumen during active inflammation may induce IL-6 secretion, which is mediated by cAMP/CREB activation and occurs in an apically polarized fashion. This would allow sequential activation of neutrophil degranulation in the lumen $-\mathrm{a}$ flow of events that would, in an epithelium-dependent fashion, enhance microbicidal activity of neutrophils as they arrive in the intestinal lumen.

J. Clin. Invest. 107:861-869 (2001).

\section{Introduction}

Adenosine is a critical extracellular signaling molecule that is produced by various cells during normal metabolic activity and released in a regulated fashion (1-3). For example, adenosine is released during inflammatory conditions and acts as a paracrine factor with diverse effects on a variety of organ systems including cardiovascular, nervous, urogenital, respiratory, and digestive systems $(2,4,5)$. Once released, adenosine interacts with an adenosine receptor belonging to the family of seven-transmembrane $G$ protein-coupled cell-surface receptors $(2,4)$. On the basis of initial pharmacological criteria, adenosine was thought to interact with one of several adenosine-receptor subtypes A1, A2a, A2b, and A3 (2, 4, 6, 7), all of which have now been cloned. One of the well-known effects of adenosine is the modulation of inflammation in many tissues through its potent and selective regulation of proinflammatory or anti-inflammatory cytokine production. For example, adenosine induces IL-10 and suppresses IL-12 secretion in monocytes (8), induces IL-8 release by mast cells (9), and stimulates IL-6 secretion by astrocytes (10).
In the intestine, neutrophil transepithelial migration and arrival in the lumen to form crypt abscesses is the pathologic hallmark of the active phase of many intestinal disorders, including inflammatory bowel disease $(1,10)$. We have shown previously that neutrophils, upon transmigration into the intestinal lumen, release $5^{\prime}$ AMP $(1,10)$. Adenosine is derived from $5^{\prime}$ AMP when it is converted by the intestinal apical membrane $5^{\prime}$ ectonucleotidase (CD 73) (11) and can subsequently interact with the intestinal adenosine receptor. In this way, as neutrophils arrive in the lumen from which a pathogenic microbial threat originates, these cells not only directly defend the surface but also stimulate a secretory flush. Other biological roles of stimulation of $\mathrm{A} 2 \mathrm{~b}$ receptors in intestinal lumen are not known. Using molecular, pharmacologic, and biochemical approaches, we characterized the intestinal adenosine receptor as the A2b subtype in both T84 cells, a model intestinal epithelial cell line, and intact human intestinal epithelia (12). Furthermore, the A2b receptor appears to be the only adenosine receptor present in T84 cells and is present in both apical and basolateral membranes (12-14). The A2b receptor is functionally cou- 
pled to Gos and stimulation of the apical or basolateral surface with adenosine results in increased cAMP (12). After preliminary observations, we have made use of the apical and basolateral expression of the A2b receptor in T84 cells to examine whether adenosine induces the secretion of IL-6, a proinflammatory cytokine that is induced by the cAMP-mediated signaling pathway, and whether this induction is polarized.

IL-6 is a 21 - to $28-\mathrm{kDa}$ glycoprotein that, under specified conditions, may be secreted by monocytes, macrophages, lymphocytes, and epithelial cells, including the intestinal epithelial cells $(15,16)$. Expression of IL- 6 is important for the host response to a number of infections, and excessive secretion of IL- 6 is thought to contribute to the pathogenesis of many diseases, including rheumatoid arthritis and inflammatory bowel disease. IL- 6 receptors are present in a variety of cells including monocyte, macrophage, lymphocyte, neutrophil, and epithelial cells (including intestinal epithelial cells) (15-17).

IL-6 is present in very high levels in both serum and intestinal tissue from patients with Crohn's disease (18-20). Il-6 has been shown to play major role in the pathogenesis of inflammatory bowel disease and is required for the development of Th1 cell-mediated colitis $(22,23)$. Intestinal epithelial cells and lamina propria mononuclear cells have been shown to be the major source of IL-6 seen in inflammatory bowel disease (21). In addition to inflammatory bowel disease, IL-6 has been shown to play a major role in the induction of B-cell differentiation, monocyte proliferation, and neutrophil recruitment to sites of inflammation (15). IL-6 has been also shown to interact with receptors on endothelial cells and decrease endothelial barrier function (24). In epithelial cells, IL-6 expression has been shown to be induced by various cytokines such as IL-1 $\beta$ (25), TNF- $\alpha(26)$, chemokines $(17,27)$, and by Salmonella typhimurium (28). It is not known if IL-6 is secreted by epithelial cells in a polarized fashion.

We demonstrate here that adenosine released in the intestinal lumen (as provided by the transmigrating neutrophils) via activation of intestinal $\mathrm{A} 2 \mathrm{~b}$ receptors causes substantial and polarized IL- 6 secretion. In contrast with secretion of many other cytokines exemplified by IL-8, IL-6 secretion is polarized to the apical compartment regardless of whether the cells are stimulated by apical or basolateral adenosine. We also show that intestinal IL-6 secretion is transcriptionally mediated by activated transcription factor (ATF) and cAMPresponsive element binding protein (CREB) elements. Finally, IL- 6 can stimulate $\left[\mathrm{Ca}^{++}\right]$signaling in neutrophils, presumably the basis for the degranulating effect of this cytokine. These data demonstrate, for the first time to our knowledge, polarized release of an epithelial proinflammatory cytokine into the luminal compartment. They also suggest that the activation of adenosine $\mathrm{A} 2 \mathrm{~b}$ receptors may provide epithelial-derived paracrine signals to neutrophils positioned apically after transmigration. Paracrine signaling of luminal immune cells provides an additional means of regulation of inflammatory responses by intestinal epithelia.

\section{Methods}

Reagents. All tissue-culture supplies were obtained from Life Technologies (Grand Island, New York, USA). Adenosine and 5 '-(N-ethylcarboxamido) adenosine (NECA) were obtained from Research Biochemicals International (Natick, Massachusetts, USA). Forskolin and carbachol were obtained from Sigma Chemical Co. (St. Louis, Missouri, USA). Reagents for SDS-PAGE and nitrocellulose membranes $(0.45-\mu \mathrm{M}$ pores) were from Bio-Rad Laboratories Inc. (Hercules, California, USA). Anti-CREB and anti-phospho-CREB were purchased from New England Biolabs Inc. (Beverly, Massachusetts, USA). Other Ab's include FITC-labeled goat anti-rabbit $\mathrm{Ab}$ and peroxidase-conjugated mouse anti-rabbit $\mathrm{Ab}$ obtained from Pierce Chemical Co. (Rockford, Illinois, USA) and New England Biolabs Inc., respectively. IL-6 and monoclonal anti-IL- 6 was obtained from R\&D Systems Inc. (Minneapolis, Minnesota, USA).

Cell culture. T84 cells (American Type Culture Collection, Rockville, Maryland, USA) were grown and maintained in culture as described previously (10) in a 1:1 mixture of DMEM and Ham's F-12 medium supplemented with penicillin $(40 \mathrm{mg} / \mathrm{l})$, ampicillin (8 $\mathrm{mg} / \mathrm{l})$, streptomycin $(90 \mathrm{mg} / \mathrm{l})$, and $5 \%$ newborn calf serum. Confluent monolayers were prepared as described previously (1). Experiments were done on cells plated for 7-8 days on permeable supports of 0.33 $\mathrm{cm}^{2}$ (inserts). Inserts with rat tail collagen-coated polycarbonate membrane filter $(5-\mu \mathrm{m}$ pore size; Corning-Costar Corp., Cambridge, Massachusetts, USA) rested in wells containing media until steady-state resistance was achieved, as described previously (1). This permits apical and basolateral membranes to be separately interfaced with apical and basolateral buffer, a configuration identical to that developed previously for various microassays (1). The T84 cells had a high electrical resistance $\left(1,200-1,500 \Omega \cdot \mathrm{cm}^{2}\right)$. All experiments were performed on T84 cells between passages 69-83. Cos-7 cells (American Type Culture Collection) were grown in DMEM containing 10\% FCS supplemented with penicillin and streptomycin.

IL- 6 assay. Cells were placed in HBSS $(250 \mu \mathrm{l}$ apical and $300 \mu \mathrm{l}$ basolateral) and apical and basolateral solution was collected at various times after stimulation and passed through $0.33-\mu \mathrm{m}$ filters to remove debris. IL-6 was measured using ELISA (Quantikine IL-6 immunoassay kit; R\&D Systems Inc.).

Plasmids, transient transfections, and chloramphenicol acetyl transferase assays. Various IL-6 promoter constructs were the generous gift of J.L. Harcourt and M.K. Offerman (Department of Medicine, Winship Cancer Center, Emory University, Atlanta, Georgia, USA) and have been characterized previously (29). The wild-type construct is the full-length IL-6 promoter -15 to -435 subcloned into pCAT3-Basic (Promega Corp., Madison, Wisconsin, USA). Mutants were gen- 
erated using wild-type IL- 6 promoter-chloramphenicol acetyl transferase (IL-6 promoter-CAT) in the NF-IL-6 site, ATF/CRE site, or NF- $\kappa B$ sites. Cells were transiently transfected using DEAE-dextran. Briefly, COS 7 cells were passaged from one p100 to four sixwell plates at a density of $2 \times 10^{5}$ cells/well for transfection the following day. The plasmid $(5 \mu \mathrm{g})$ was diluted in $380 \mu \mathrm{l}$ of $10 \mathrm{mM}$ Tris- $\mathrm{HCl}, \mathrm{pH} 7.5$, containing $100 \mathrm{mM} \mathrm{NaCl}$. Twenty microliters of DEAE-dextran $(10 \mathrm{mg} / \mathrm{ml}$ in PBS) was added to this mixture. Cells were washed with PBS, dextran-DNA mixture was added to the cells, and they were incubated at $37^{\circ} \mathrm{C}$ for 30 minutes. Serum-free media containing 0.2 $\mu \mathrm{M}$ chloroquine was added to the cells, and after 2.5 hours of incubation at $37^{\circ} \mathrm{C}$, cells were washed with $10 \%$ DMSO in serum-free media. Cells were then washed thoroughly with PBS, incubated for 66 hours in regular media, and subsequently stimulated for 4 hours with adenosine. CAT assays were performed according to manufacturer's protocol (Promega-CAT assay kit). Cells were lysed and protein quantitation was done according to either the Bradford or Lowry assay kit (Bio-Rad Laboratories Inc.). The cell viability was not impaired by transfection or other aspects of these treatments as judged by cell survival.

Confocal microscopy. Monolayers of T84 cells were washed in HBSS, fixed in buffered formaldehyde for 20 minutes, permeabilized with saponin, incubated with respective primary Ab's overnight in a humidity chamber, washed with HBSS, and subsequently incubated with fluoresceinated secondary Ab's (Jackson ImmunoResearch Laboratories, West Grove, Pennsylvania, USA). Monolayers, mounted in $p$-phenylenediamine glycerol $(1: 1)$, were analyzed by confocal microscopy (Zeiss dual laser confocal microscope; Carl Zeiss Inc., Thornwood, New York, USA).

SDS-PAGE and Western blot. T84 cells were lysed with PBS containing $1 \%$ Triton X-100 and 1\% Nonidet P-40 (NP-40; $\mathrm{vol} / \mathrm{vol}$ ), protease-inhibitor cocktail, EDTA, SDS, $1 \mathrm{mM}$ sodium orthovanadate, and $1 \mathrm{mM}$ sodium fluoride. SDSPAGE was performed according to the Laemmli procedure using 10\% acrylamide gel. Proteins were electrotransferred to nitrocellulose membranes and probed with anti-CREB or anti-phospho-CREB Ab's (diluted 1:1,000). Subsequently, membranes were incubated with the corresponding peroxidase-linked secondary $\mathrm{Ab}$ diluted 1:2,000, washed, and were subsequently incubated with enhanced chemiluminescence (ECL) reagents (Amersham Pharmacia Biotech, Piscataway, New Jersey, USA) before exposure to high-performance chemiluminescence films (Amersham Pharmacia Biotech). For mol wt determination, polyacrylamide gels were calibrated using standard proteins (Bio-Rad Laboratories Inc.) with mol wt markers within the range 7,700 to 214,000 .

Neutrophil isolation. Polymorphonuclear neutrophils (PMNs) were isolated from whole blood (anticoagulated with citrate/dextrose) obtained from healthy volunteers, using a gelatin sedimentation technique described previously (10). PMNs were resuspended in modified HBSS devoid of $\left[\mathrm{Ca}^{++}\right]$and $\mathrm{Mg}^{2+}(\mathrm{HBSS})$ at a concentration of $4 \times 10^{7}$ cells $/ \mathrm{mL}\left(4^{\circ} \mathrm{C}\right)$ and used for subsequent experiments.

Determination of intracellular $\left[\mathrm{Ca}^{++}\right]$. Intracellular $\left[\mathrm{Ca}^{++}\right]$ was determined as described previously (30). Neutrophils were loaded with Indo-I and placed into a spectrofluorometer $\left(\right.$ at $\left.37^{\circ} \mathrm{C}\right)$. Fluorescence excitation was read at $355 \mathrm{~nm}$ while the excitation wavelength was alternated between 405 and $485 \mathrm{~nm}$ four times per second via Intracellular Cation Software (Hitachi, Sunnyvale, California, USA). After reading fluorescence for 3 minutes, fMLP, IL- $6 \pm$ IL- 6 Ab was added, as indicated in the figure legends. Values of intracellular $\left[\mathrm{Ca}^{++}\right]$were calculated as described previously (30).

\section{Results}

Adenosine induces polarized secretion of IL-6 mediated by the $A 2 b$ receptor. Adenosine added either to the apical or basolateral side of T84 cells elicited IL-6 secretion that was apically polarized to the apical side, irrespective of the side of stimulation. As shown in Figure 1a, adenosine $(100 \mu \mathrm{M})$ added to the apical compartment of T84 cells induced approximately a ninefold increase in IL-6 secretion compared with T84 cells treated with vehicle alone. In contrast, there was no significant elevation of

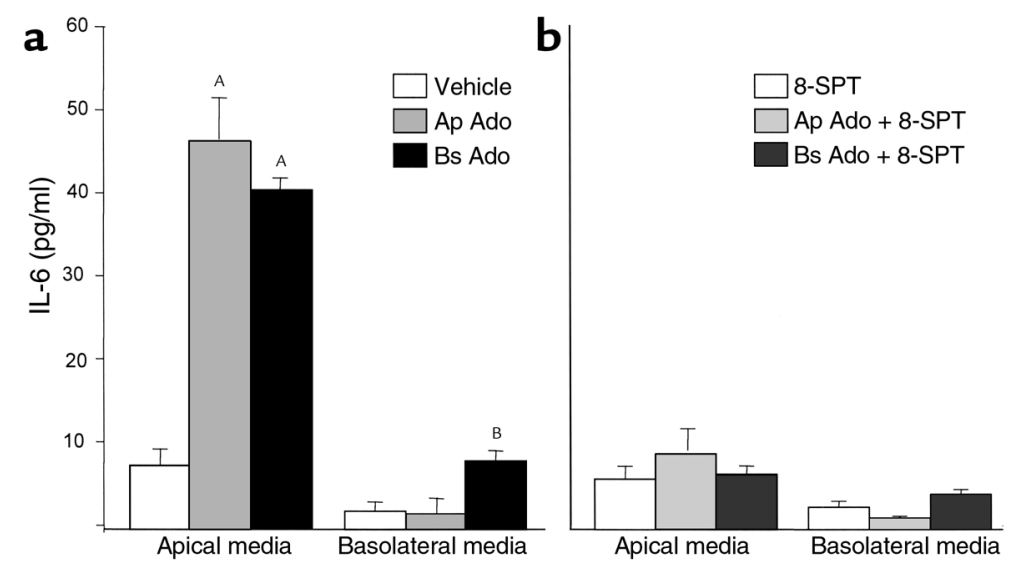

Figure 1

IL-6 induction by adenosine. T84 monolayers were prewashed in HBSS and equilibrated at $37^{\circ} \mathrm{C}$ for 20 minutes. Adenosine (Ado; $100 \mu \mathrm{M}$ ) was added to the apical (Ap) or basolateral (Bs) compartment. After an incubation period of 5.5-6 hours at $37^{\circ} \mathrm{C}$, IL- 6 was measured in the apical or basolateral compartment as described in Methods. Values are expressed as $\mathrm{pg} / \mathrm{ml}$. Data represent the responses observed in three separate experiments plotted as mean $\pm \mathrm{SD}, n=3$ samples per treatment group. Footnotes represent values significantly different from monolayers treated with vehicle alone, ${ }^{A} P<0.001,{ }^{B} P<0.05$. (a) Monolayers treated with either vehicle or adenosine. (b) Monolayers treated with either adenosine $(100 \mu \mathrm{M})$ or adenosine plus 8-SPT $(100 \mu \mathrm{M})$. Ap, apical; Bs, basolateral. 
Table 1

IL-6 secretion: dose response to adenosine

\begin{tabular}{lcccc} 
& \multicolumn{2}{c}{ Ap adenosine } & \multicolumn{2}{c}{ Bs adenosine } \\
& Ap media & Bs media & Ap media & Bs media \\
No addition & $3.0 \pm 0.4$ & $2.0 \pm 0.2$ & $3.6 \pm 0.8$ & $0.9 \pm 0.6$ \\
$0.01 \mu \mathrm{M}$ & $3.6 \pm 1.0$ & $0.9 \pm 0.1$ & $3.9 \pm 0.2$ & $1.2 \pm 0.4$ \\
$0.1 \mu \mathrm{M}$ & $3.4 \pm 1.2$ & $1.5 \pm 0.1$ & $3.7 \pm 0.5$ & $1.1 \pm 0.7$ \\
$1.0 \mu \mathrm{M}$ & $8.4 \pm 2.0^{\mathrm{A}}$ & $1.8 \pm 0.4$ & $5.2 \pm 1.0$ & $1.9 \pm 0.2$ \\
$10 \mu \mathrm{M}$ & $27.0 \pm 3.0^{\mathrm{B}}$ & $2.0 \pm 0.6$ & $10.9 \pm 1.9^{\mathrm{A}}$ & $3.2 \pm 0.3^{\mathrm{A}}$ \\
$100 \mu \mathrm{M}$ & $30.0 \pm 3.4^{\mathrm{B}}$ & $4.5 \pm 1.5$ & $29.6 \pm 3.2^{\mathrm{B}}$ & $3.1 \pm 1.3^{\mathrm{A}}$
\end{tabular}

IL-6 in picograms per milliliter. Significantly different from controls, ${ }^{A} P<0.05$; B $P<0.001$. Ap, apical; Bs, basolateral.

IL-6 levels in the basolateral media. We have shown previously that basolaterally secreted cytokines are readily measured by such approaches (30). Similar polarized secretion of IL- 6 was obtained when the T84 cells were stimulated with basolateral adenosine. IL- 6 in the basolateral compartment was mildly but significantly increased upon stimulation with basolateral adenosine.

We next studied the effect of 8 -( $p$-sulfophenyl theophylline) (8-SPT), an adenosine-receptor antagonist, on the IL- 6 secretion induced by adenosine. As seen in Figure $1 b$, IL- 6 secretion induced by apical or basolateral treatment of T84 cells with adenosine was abolished when cells were also exposed to 8-SPT $(100 \mu \mathrm{M})$ at the time of adenosine stimulation, suggesting that the IL-6 secretion is mediated by the adenosine receptor.

Adenosine induces IL-6 secretion in a dose-dependent fashion. A dose response of IL- 6 secretion to adenosine is shown in Table 1. Apical adenosine induced significant IL- 6 secretion at a dose of $1 \mu \mathrm{M}$, while basolateral adenosine induced significant IL- 6 secretion only at higher concentrations. The rightward shift of the dose response to basolateral adenosine is consistent with our previous observation and is due to the presence of adenosine deaminase on the basolateral membrane of the epithelial cells (12). The addition of apical or basolateral NECA, a nonmetabolized analogue of adenosine, induced an identical doseresponse curve (data not shown).

Time course of IL- 6 induction by adenosine. The time course of induction of IL- 6 by adenosine (Figure 2) showed that significant IL- 6 secretion into the apical media occurred 3 hours after exposure to adenosine with maximal secretion at 6 hours. Apical and basolateral adenosine induced a twofold increase in IL-6 secretion in the apical media at 3 hours, with maximal stimulation (ninefold to tenfold increase) between 3 and 6 hours after adenosine stimulation. We also performed experiments to determine the length of exposure to adenosine required to induce maximal IL- 6 secretion. To do this, cells were exposed to adenosine for 10,60 , 120 minutes, washed, and apical and basolateral media were collected for a total of 6 hours. Our results showed that a 10 -minute exposure to adenosine was sufficient to induce maximal IL-6 secretion (data not shown).

Stimulation of IL- 6 by other stimuli is polarized. To see if IL- 6 secretion is polarized after stimulation by other proinflammatory stimuli, T84 cells were stimulated with S. typhimurium and TNF- $\alpha$ (both of which have been shown to induce IL- 6 transcripts in intestinal epithelial cells) and forskolin, a direct activator of adenylate cyclase (28). As shown in Figure 3, forskolin $(10 \mu \mathrm{M})$, S. typhimurium, and TNF- $\alpha(100 \mathrm{ng} / \mathrm{ml})$ induced a greater than tenfold increase in IL-6 secretion, which also was polarized to the apical compartment. All of these stimuli also increased IL- 6 secretion in the basolateral compartment albeit to much lesser extent (vehicle, forskolin, S. typhimurium, and TNF- $\alpha$ respectively, in picograms per milliliter: $1.9 \pm 0.5,4.9$ $\pm 0.4,12.1 \pm 4.1,33.4 \pm 4.2)$. Carbachol, an agent that increases intracellular $\left[\mathrm{Ca}^{++}\right]$, did not increase IL-6 secretion in the apical or the basolateral media.

Adenosine-induced IL-6 secretion is transcriptionally mediated. IL- 6 promoter activity has been localized to -1 to -435 upstream of the start site. The IL- 6 promoter contains two overlapping regions - second messenger (also called multiple responsive elements or MREs) and cytokine responsive element - defined by their roles in IL-6 induction by a variety of inducers $(15,29)$ (Figure 4a). Critical consensus elements within the MREs have been identified. MRE I contains an ATF/CREB site and MRE II contains a NF-IL-6 site (also called c/EBP $\beta$ ). Agents that increase intracellular cAMP, as adenosine and forskolin are known to do, induce IL-6 secretion by the activation of both MRE I and MRE II. Additional sites that are important for enhanced IL- 6 expression in response to various inducers include the AP- 1 site $(-283$ to -277$)$ and the NF- $\kappa \mathrm{B}$ site $(-73$ to -63$)$. In addition, the IL- 6 promoter also contains several trans-acting elements that downregulate IL- 6 transcription, such as the glucocorticoid responsive element and IFN regulatory factor.

We studied the effect of adenosine on the induction of the IL- 6 promoter linked to the CAT reporter gene.

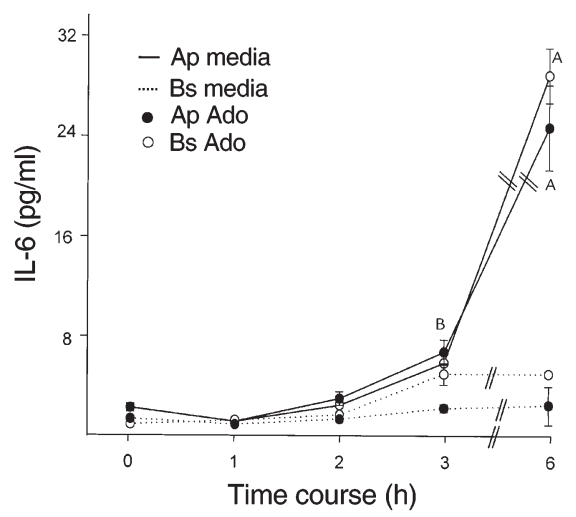

\section{Figure 2}

Time course of IL- 6 secretion. T84 monolayers were prewashed in HBSS. After equilibration at $37^{\circ} \mathrm{C}$ for 20 minutes, cells were stimulated with adenosine (apical or basolateral; $100 \mu \mathrm{M}$ ), HBSS was collected for 1, 2, 3, or 6 hours, and IL-6 was measured as described in Methods. Values are expressed as $\mathrm{pg} / \mathrm{ml} \mathrm{IL}-6$. Data represent the responses observed in three separate experiments plotted as mean $\pm \mathrm{SD}, n=2$ samples per treatment group. Footnotes represent values significantly different from the respective 0 time point, ${ }^{A} P<0.001,{ }^{B} P<0.05$. 


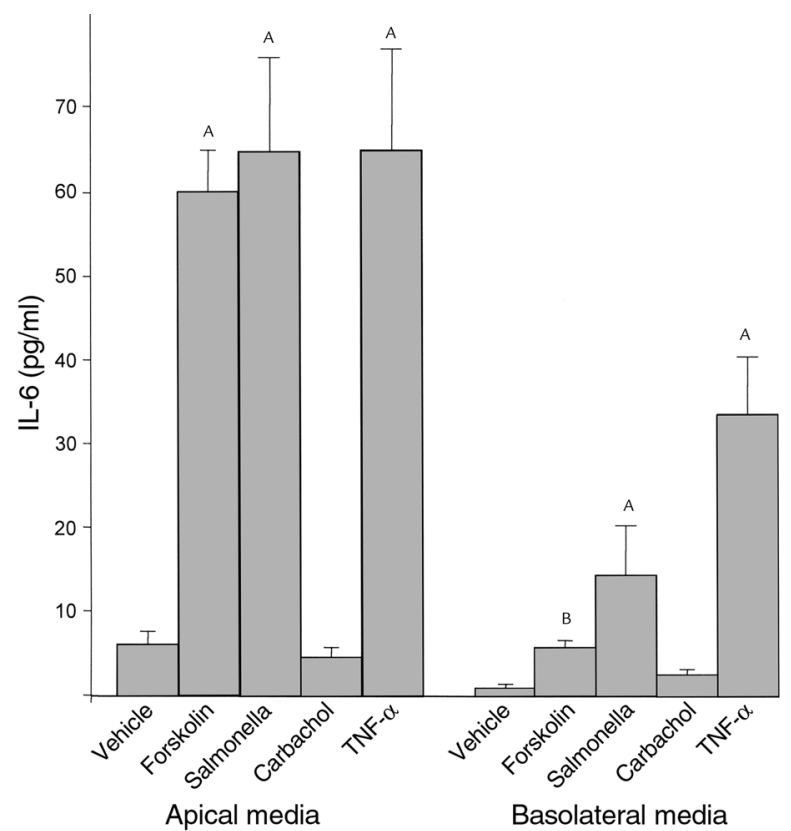

Figure 3

Effect of various proinflammatory agents on IL-6 secretion. T84 cells were washed with $\mathrm{HBSS}$. After equilibration of 20 minutes at $37^{\circ} \mathrm{C}$, forskolin $(10 \mu \mathrm{M})$, TNF- $\alpha(100 \mathrm{ng} / \mathrm{ml})$, and carbachol $(1 \mu \mathrm{M})$ were added basolaterally. Apical and basolateral media were collected for 5.5-6 hours after stimulation. S. typhimurium was added apically for 1 hour for surface colonization (30), after which the monolayers were rinsed thoroughly with HBSS and incubated for an additional 5 hours, and IL-6 assays were performed. Data represent the responses observed in two separate experiments plotted as mean $\pm S D, n=2$ per treatment group. ${ }^{A}$ Significantly different from the unstimulated control, $P<0.001$. BSignificantly different from unstimulated control, $P<0.05$

COS-7 cells that possess A2b receptors were used in view of the difficulty of transfecting T84 cells. It has been shown previously that the A2b receptors in COS7 cells, like those in the T84 cells, positively couple to adenylate cyclase (31). Stimulation of COS-7 cells with adenosine thus results in increased levels of intracellular cAMP, and this forms the major signaling pathway induced by adenosine via the $\mathrm{A} 2 \mathrm{~b}$ receptor. In addition, COS 7 cells secrete IL-6 (unstimulated: $36.8 \pm 3.5$; adenosine $100 \mu \mathrm{M}$ : $155.5 \pm 25.0 \mathrm{pg} / \mathrm{ml})$. As seen in Figure $4 \mathrm{~b}$, adenosine induced approximately a 40 -fold increase in CAT activity in the cells transfected with the full-length IL-6 promoter construct (+15 to -435$)$. Mutations in either the region of the ATF/CREB or NF-IL-6 elements of the IL- 6 promoter abolished responsiveness to adenosine (Figure $4 \mathrm{~b}$ ) while mutation of the NF- $\mathrm{KB}$ site did not affect the CAT activity in response to adenosine. Such data imply that both the ATF/CREB and the NF-IL- 6 sites are important for adenosine induced IL- 6 secretion.

Activation of ATF/CREB mediates adenosine-induced IL-6 secretion. CREB and ATF are nuclear transcription factors that are activated by cAMP-mediated signaling pathways. Since the only known signaling molecule induced by adenosine is cAMP, we next sought to see if adenosine induced activation of CREB in T84 cells. As seen in Figure 5, apical or basolateral stimulation with adenosine $(100 \mu \mathrm{M})$ resulted in phosphorylation of CREB while the total CREB remained unchanged. Using Ab's to phosphorylated CREB at ser-133, we showed that adenosine induced phosphorylated CREB 5 minutes after stimulation, and maximal induction was seen at 1 hour after stimulation. In addition, both apical and basolateral adenosine induced phosphorylated ATF-1, a transcription factor that belongs to the CREB family. The phosphorylated ATF-1, like CREB, was detected at 5 minutes after adenosine stimulation with maximal levels seen at 1 hour (Figure 5). Both the phosphorylated CREB and ATF-1 induction returned to baseline by 6 hours (Figure 5).

To study the cellular localization of phosphorylated CREB, cells were stimulated with adenosine for 5,45 , or 60 minutes and subsequently stained for immunolocalization of CREB. While control cells showed no
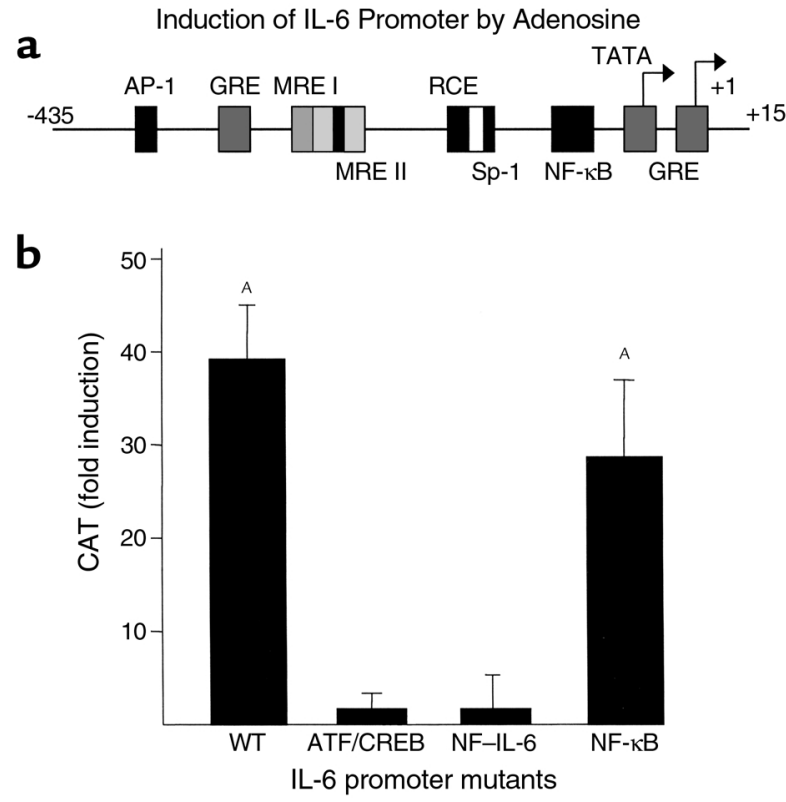

Figure 4

Effect of adenosine on IL-6 promoter activity and analysis of adenosine-mediated regulation of IL-6 promoter mutants. (a) Most elements of the IL- 6 promoter that have been characterized lie within the 300 bp proximal to the start site $(+1)$ of transcription. These elements include multiple AP-1-binding sites, an IRF-1-binding site, three glucocorticoid response elements (GRE), two second-messenger and cytokine-responsive elements (MREs), the first of which contains ATF/CREB binding sites and the second a binding site for $\mathrm{C} / \mathrm{EBP} \beta$ or NF-IL-6-binding site, and a NF- $\mathrm{KB}$-binding element. The promoter also contains two transcription start sites, one major and one minor. Mutants that were generated in the ATF/CRE, NF-IL-6, and NF- $\mathrm{KB}$ sites are indicated in Methods. (b) COS-7 cells were transiently transfected with wild-type (WT) or various mutant IL-6 CAT constructs. Cells were stimulated with adenosine 66 hours after transfection, and a CAT assay was performed 4 hours after stimulation. Data represent fold increase over transfected and unstimulated control (expressed as nanograms CAT/ $\mu$ g protein) observed in two separate experiments plotted as mean \pm SD from duplicate determination of two samples per group, $n=2$. 


\section{Figure 5}

Expression of phosphorylated ATF/CREB in response to adenosine. T84 cells were washed with HBSS, equilibrated for 20 minutes at $37^{\circ} \mathrm{C}$, and stimulated with adenosine $(100 \mu \mathrm{M}$, apical or basolateral) for the various times indicated. Whole-cell detergent lysates (approximately $15 \mu \mathrm{g}$ protein/lane) were resolved by SDS-PAGE and immunoblotted for phosphorylated ATF/CREB (a) and total CREB (b). Scanning densitometry of the blot is shown in $\mathbf{b}$ depicting a four- and eightfold increase in ATF-1 and CREB, respectively compared with 0 time. The induction was maximal at 60 minutes and declined at 180 and 360 minutes after stimulation. Data represent the responses observed in two separate experiments with two filters per time point.

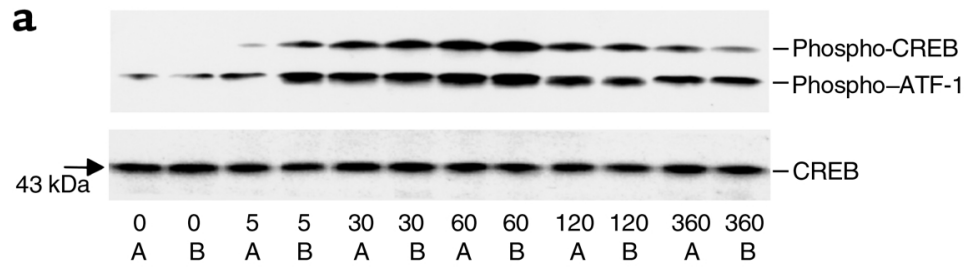

b
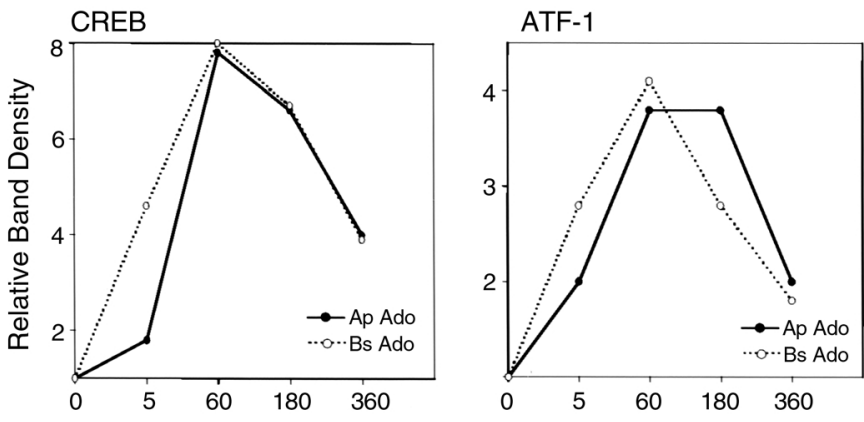

staining of phosphorylated CREB at any of the time points tested, cells treated with apical or basolateral adenosine for 5 minutes showed nuclear staining of phosphorylated CREB (Figure 6, a, b, and c). Control cells are outlined with rhodamine-phalloidin staining in Figure 6a. Similar results were obtained at 30 and 60 minutes after adenosine stimulation (data not shown).

IL-6 induces neutrophil activation. Transmigrated neutrophils can release 5'AMP into the lumen (10), which is converted to adenosine (11) and recognized by the apical A2b receptors on intestinal epithelia (12). Apical adenosine stimulation, as shown above, stimulates apical IL-6 secretion. Thus, we next sought to determine if IL-6 might, in turn, modify neutrophil responses, thus indicating potential of neutrophil-toepithelial cell and epithelial-to-neutrophil paracrine loops after transepithelial migration. Because calcium mobilization is part of many of the pathways by which neutrophils are activated, we measured intracellular $\left[\mathrm{Ca}^{++}\right]$flux in response to graded doses of IL-6 (25-100 $\mathrm{pg} / \mathrm{ml})$ with or without anti-IL-6 mAb. As seen in Figure 7, IL-6 increased intracellular $\left[\mathrm{Ca}^{++}\right]$in neutrophils in a dose-dependent fashion. IL-6 $(100 \mathrm{pg} / \mathrm{ml})$ induced a $\left[\mathrm{Ca}^{++}\right]$ flux that was comparable to that induced by $\mathrm{MLP}\left(10^{-5} \mathrm{M}\right)$. The effect of IL- 6 on the $\left[\mathrm{Ca}^{++}\right]$ flux was completely abolished by the anti-IL- $6 \mathrm{mAb}$, thus indicating that $\left[\mathrm{Ca}^{++}\right]$mobilization is due to IL-6. Thus these data demonstrate a paracrine loop in which there is cross-talk between epithelial cells and neutrophils after neutrophil transmigration.
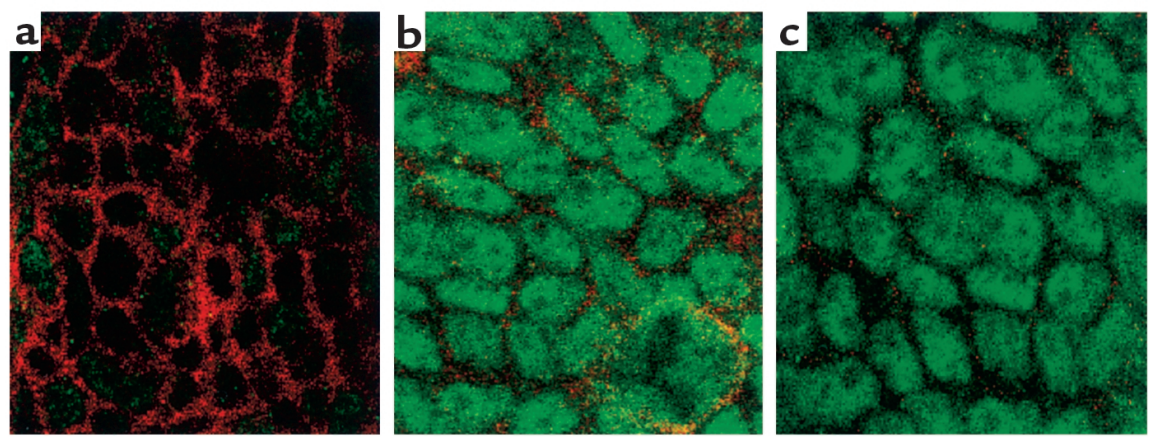

\section{Figure 6}

Confocal microscopy of phospho-CREB. T84 monolayers were incubated with apical or basolateral adenosine $(100 \mu \mathrm{M})$ for 5 minutes. Nuclear staining was determined by immunofluorescence labeling and confocal microscopy. The en face images document the presence of activated phospho-CREB staining in cells treated with apical or basolateral adenosine (b and $\mathbf{c}$, respectively). Control cells double-stained with phospho-CREB and rhodamine-phalloidin are shown in a. Similar results were obtained at 30 and 60 minutes after adenosine stimulation. 


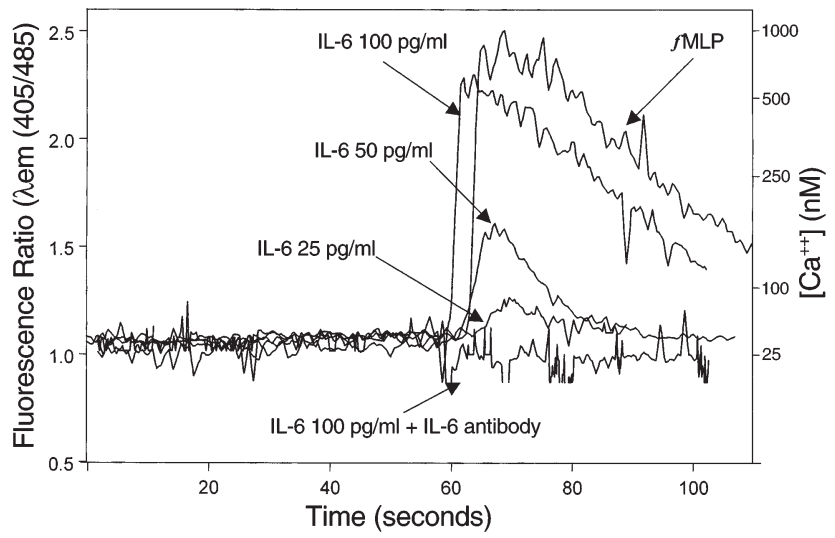

Figure 7

Effect of IL-6 on neutrophil calcium response. Neutrophils $\left(10^{6}\right)$ were loaded with the $\left[\mathrm{Ca}^{++}\right]$indicator Indo-1 and placed in a thermostated spectrofluorometer, and intracellular $\left[\mathrm{Ca}^{++}\right]$was measured as described in Methods. Stimuli were added at 60 seconds. Intracellu$\operatorname{lar}\left[\mathrm{Ca}^{++}\right]$was measured in response to $\mathrm{PMLP}\left(10^{-6} \mathrm{M}\right), \mathrm{IL}-6$ in the indicated doses, and monoclonal anti-IL- 6 added along with IL-6 $(100 \mathrm{pg} / \mathrm{ml})(\mathrm{IL}-6+\mathrm{IL}-6 \mathrm{Ab})$.

induce IL-6 secretion predominantly in the apical compartment. Moreover, baseline IL-6 secretion in unstimulated epithelial monolayer is also significantly higher apically than basolaterally. Similar apically polarized secretion has been demonstrated recently for secretory leukocyte protease inhibitor (SLPI) in intestinal epithelial cells (32). The induction of basolateral IL-6 secretion, though still far less than apical secretion, is significant following TNF- $\alpha$ stimulation. One explanation for apparent basolateral IL-6 secretion in response to TNF- $\alpha$ is related to the drop in barrier function (transepithelial resistance), which also occurs in response to TNF- $\alpha$ (33) and likely diminishes the ability to rapidly separate apical and basolateral compartments.

The induction of IL- 6 by adenosine is likely mediated by the $\mathrm{A} 2 \mathrm{~b}$ receptor (the only adenosine receptor subtype present in this cell line) because 8-SPT, an adenosine-receptor antagonist, abolishes IL- 6 induction by both apical and basolateral adenosine. The dose response of adenosine-induced IL-6 secretion is also suggestive of a receptor-mediated process with satura- tion of IL- 6 induction seen at $10 \mu \mathrm{M}$ for apical adenosine and $100 \mu \mathrm{M}$ for basolateral adenosine. The IL-6 induction by adenosine appears to be a transcriptionmediated process and not release of preformed or stored IL-6, since IL-6 induction is seen 3 hours after stimulation with adenosine. Consistent with this notion is our data on the transcriptional activation of IL- 6 promoter by adenosine. We demonstrate that adenosine induces the activation of the full-length IL- 6 promoter and the ATF/CREB and NF-IL- 6 sites, but not the NF- $\mathrm{\kappa B}$ site of the IL-6 promoter.

We have shown previously that the only demonstrable signaling pathway for adenosine is mediated by cAMP/protein kinase A (PKA), and adenosine does not induce calcium or PKC activity (ref. 12; S.V. Sitaraman and J.L. Madara, unpublished observation). Adenosine induces a polarized increase in cAMP in the intestinal epithelial cells. While basolateral adenosine stimulation results in several-fold increase in cAMP, apical stimulation results in small but significant increase in cAMP in response to the same dose of adenosine (12). We demonstrate here that adenosine-induced IL- 6 secretion is mediated by a time-dependent activation of CREB, a nuclear transcription factor whose activation by the cAMP/PKA pathway is well established (34). Interestingly, despite disparate cAMP levels induced by apical versus basolateral adenosine, the kinetics of CREB induction and the phosphorylation of nuclear CREB did not differ. This suggests that the signaling at the apical membrane perhaps occurs in microdomains that contain the $\mathrm{A} 2 \mathrm{~b}$ receptor and its signaling effectors.

The kinetics of IL- 6 induction shows that a 10minute exposure to adenosine is sufficient for maximal IL-6 induction, suggesting that the signal transduction process is complete within this time frame. Consistent with this notion is the phosphorylation of CREB, which occurs by 5 minutes. Based on our observation that the only known signaling pathway for adenosine in T84 cells is cAMP/PKA mediated and that adenosine induces a time-dependent activation of phosphorylated CREB and its nuclear translocation in T84 cells, we conclude that adenosine induces IL- 6 via transcriptional activation mediated by cAMP. This is also supported by the inability of carbachol, an agent that increases intracellular $\left[\mathrm{Ca}^{++}\right]$, to induce IL-6.

\section{Figure 8}

Schematic representation of epithelial-neutrophil interaction in a crypt abscess. An epithelial monolayer with neutrophils transmigrating to the luminal compartment is shown at the left. Inset shows that adenosine is derived from the enzymatic conversion (mediated by epithelial ectonucleotidase, CD 73) of $5^{\prime}$ AMP released in the lumen by the neutrophils. Adenosine thus released interacts with the adenosine $\mathrm{A} 2 \mathrm{~b}$ receptor, a $\mathrm{G}$ protein-coupled receptor, resulting in an increase in intracellular cAMP that may be involved in the transcriptional activation of IL-6 secretion. IL-6 is preferentially released in the apical compartment and induces intracellular $\left[\mathrm{Ca}^{++}\right]$flux in neutrophils, which may be involved in the release of oxygen radicals, elastase, etc., from the neutrophils.

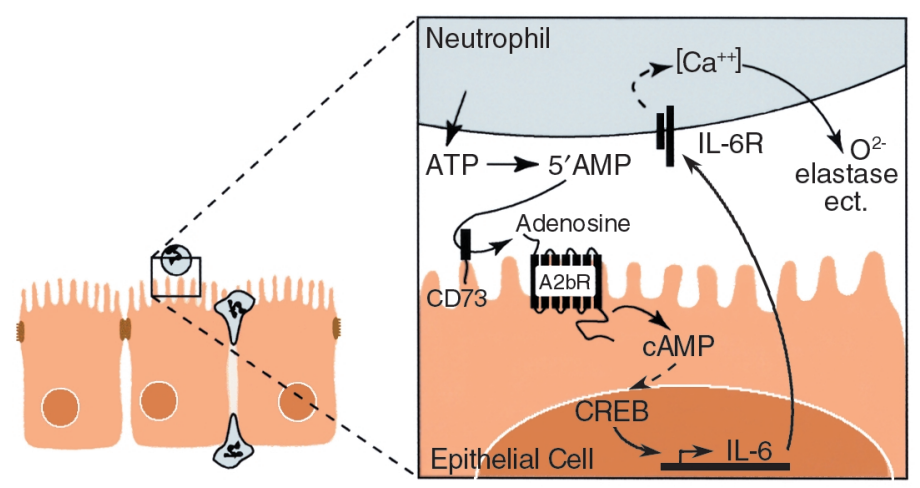


CREB and ATF are nuclear transcription factors that are activated upon phosphorylation of Ser-133 residue in the PKA-inducible domain (34). NF-IL-6 is distinct from the CREB family of transcription factors and is activated by CAMP and calcium-mediated signaling pathways (10). The promoter region of NF-IL-6 has CRE and is induced by cAMP-mediated signaling pathways (35). Activated NF-IL-6 forms heterodimers with activated ATF/CREB to bind to the promoter region of target genes (16). Therefore, the cAMP-mediated signaling pathway may be a common mechanism for the activation of IL- 6 transcription through CRE and NF-IL-6 elements by adenosine. This is consistent with the observation by others that CRE and NF-IL- 6 are the major sites for transcriptional activation of IL- 6 induced by agents that increase intracellular cAMP such as forskolin and cholera toxin $(10,36)$.

IL-6 is an important proinflammatory cytokine that is consistently seen in high levels in the serum and tissue of patients with active inflammatory bowel disease, and one of the sources for the IL- 6 is the lamina propria cells, including macrophages and monocytes (18-20,37). In this article we provide further evidence that the epithelial cell production of chemokines may also contribute to immune regulation at the mucosal surface. Here we have shown that IL- 6 is secreted into the luminal compartment. In considering the potential role of this cytokine, neutrophils seem a logical target, not only because they transmigrate into the lumen during active inflammation, but because they also provide paracrine apical adenosine signals and possess IL-6 receptors (15). Our data suggest that apically secreted IL- 6 may indeed increase neutrophil intracellular $\left[\mathrm{Ca}^{++}\right]$in neutrophils - a classic early signal in neutrophil activation. This specific signaling event is consistent with studies of downstream events in which IL-6 exposure has been shown to induce elastase release, PAF production, and production of oxygen-free radicals by neutrophils (38). IL-6 has also been shown to interact with intestinal epithelial cells via IL-6 receptors $(18,27)$. For example, IL-6 induces the sodium-glucose cotransporter in the small intestinal cells causing an increased uptake of glucose (39). The other potential effect of IL-6 on epithelial cells is being explored currently in our laboratory.

In conclusion, we believe this is the first demonstration of apically polarized cytokine secretion by intestinal epithelial cells in response to an effector, here adenosine, an endogenous nucleoside that is generated in situ during active inflammation. The effect of epithelial-derived IL-6 on induction of intracellular $\left[\mathrm{Ca}^{++}\right]$in neutrophils strongly suggests that this cytokine plays a relevant physiological role in the interaction between the epithelial cells and neutrophils (Figure 8). Future studies will involve confirming our in vitro observation in natural human intestinal epithelia. Taken together with its effect on chloride secretion, the event underlying secretory diarrhea, adenosine thus may act as a proinflammatory agent, and adenosine antagonists may therefore be useful agents to ameliorate particular aspects of intestinal inflammation such as IL-6-mediated neutrophil activation in the crypt lumen.

\section{Acknowledgments}

This work was supported by NIH grant K08 DK-02802 (to S. Sitaraman), CCFA career development awards (to S.V. Sitaraman and D. Merlin), and NIH grants K01 DK-02792 (to A.T. Gewirtz), and DK-35932 and DK47662 (to J.L. Madara). We would like to thank Michael Hobart for his excellent graphic art and Anjali Rao for a critical reading of the manuscript.

1. Nash, S., Parkos, C., Nusrat, A., Delp, C., and Madara, J.L. 1991. In vitro model of intestinal crypt abscess: a novel neutrophil-derived secretagogue (NDS) activity. J. Clin. Invest. 87:1474-1477.

2. Palmer, T.M., and Stiles, G.L. 1995. Adenosine receptors. Neuropharmacology. 34:683-694.

3. Roman, R.M., and Fitz, J.G. 1999. Emerging roles of purinergic signalling in gastrointestinal epithelial secretion and hepatobiliary function. Gastroenterology. 116:964-979.

4. Ralevic, V., and Burnstock, G. 1998. Receptors for purines and pyrimidines. Pharmacol. Rev. 50:413-492.

5. Van Belle, H., Gossens, F., and Wynants, J. 1987. Formation and release of purine catabolites during hypoperfusion, anoxia, and ischemia. Am. J. Physiol. 252:H886.

6. Feoktistov, I., and Biaggioni, I. 1997. Adenosine A2B receptors. Pharmacol. Rev. 49:381-402.

7. Linden, J., Auchampach, J.A., Xiaowei, J., and Figler, R.A. 1995. The structure and function of $\mathrm{A} 1$ and $\mathrm{A} 2 \mathrm{~B}$ adenosine receptors. Life Sci. 62:1519-1524.

8. Link, A.A., et al. 2000. Ligand-activation of adenosine A2a receptors inhibits IL-12 production by human monocytes. J. Immunol. 164:436-442.

9. Feoktistov, I, and Biaggioni, I. 1995. Adenosine A2b receptors evoke interleukin-8 secretion in human mast cells. An enprofylline-sensitive mechanism with implications for asthma. J. Clin. Invest. 96:1979-1986.

10. Madara, J.L., et al. 1993. 5'AMP is the neutrophil-derived paracrine factor that elicits chloride secretion from T84 intestinal epithelial monolayers. J. Clin. Invest. 91:5716-5723.

11. Strohmeier, G.R., et al. 1997. Surface expression, polarization, and functional significance of CD73 in human intestinal epithelia. J. Clin. Invest. 99:2588-2601.

12. Strohmeier, G.R., Reppert, S.M., Lencer, W.I., and Madara, J.L. 1995. The $\mathrm{A} 2 \mathrm{~b}$ adenosine receptor mediates cAMP responses to adenosine receptor agonists in human intestinal epithelia. J. Biol. Chem. 270:2387-2394.

13. Barrett, K.E., Cohn, J.A., Huott, P.A., Wasserman, S.I., and Dharmsathaphorn, K. 1990. Immune-related intestinal chloride secretion. II. Effect of adenosine on T84 cell line. Am. J. Physiol. 258:C902-C912.

14. Sitaraman, S.V., Si-Tahar, M., Merlin, D., Strohmeier, G.R., and Madara, J.L. 2000. Polarity of A2b adenosine receptor expression determines characteristics of receptor desensitization. Am. J. Physiol. Cell. Physiol. 278:C1230-C1236.

15. Keller, E.T., Wanagat, J., and Ershler, W.B. 1996. Molecular and cellular biology of interleukin-6 and its receptor. Front. Biosci. 1:d340-d357.

16. Simpson, R.J., Hammacher, A., Smith, D.K., Matthews, J.M., and Ward, L.D. 1997. Interleukin-6: structure-function relationships. Protein Sci. 6:929-955.

17. Shirota, K., LeDuy, L., Yuan, S.Y., and Jothy, S. 1990. Interleukin-6 and its receptor are expressed in human intestinal epithelial cells. Virchows Arch. B Cell Pathol. Incl. Mol. Pathol. 58:303-308.

18. Hosokawa, T., et al. 1999. Interleukin-6 and soluble interleukin-6 receptor in the colonic mucosa of inflammatory bowel disease. J. Gastroenterol. Hepatol. 14:987-996.

19. Reinisch, W., et al. 1999. Clinical relevance of serum interleukin-6 in Crohn's disease: single point measurements, therapy monitoring, and prediction of clinical relapse. Am. J. Gastroenterol. 94:2156-2164.

20. Louis, E., et al. 1997. A high serum concentration of interleukin-6 is predictive of relapse in quiescent Crohn's disease. Eur. J. Gastroenterol. Hepatol. 9:939-944.

21. Kusugami, K., et al. 1995. Elevation of interleukin-6 in inflammatory bowel disease is macrophage- and epithelial cell-dependent. Dig. Dis. Sci. 40:949-959.

22. Atreya, R., et al. 2000. Blockade of interleukin 6 trans signaling suppresses T-cell resistance against apoptosis in chronic intestinal inflam- 
mation: evidence in crohn disease and experimental colitis in vivo. Nat. Med. 6:583-588.

23. Yamamoto, M., Yoshizaki, K., Kishimoto, T., and Ito, H. 2000. IL-6 is required for the development of Th1 cell-mediated murine colitis. J. Immunol. 164:4878-4882.

24. Yan, S.F., Ogawa, S., Stern, D.M., and Pinsky, D.J. 1997. Hypoxiainduced modulation of endothelial cell properties: regulation of barrier function and expression of interleukin-6. Kidney Int. 51:419-425.

25. Moon, M.R., et al. 2000. Interleukin-1beta induces complement component C 3 and IL- 6 production at the basolateral and apical membranes in a human intestinal epithelial cell line. Shock. 13:374-378.

26. McGee, D.W., Beagley, K.W., Aicher, W.K., and McGhee, J.R. 1993. Transforming growth factor-beta and IL-1 beta act in synergy to enhance IL-6 secretion by the intestinal epithelial cell line, IEC-6. J. Immunol. 151:970-978.

27. Molmenti, E.P., Ziambaras, T., and Perlmutter, D.H. 1993. Evidence for an acute phase response in human intestinal epithelial cells. J. Biol. Chem. 268:14116-14124.

28. Weinstein, D.L., O’Neill, B.L., and Metcalf, E.S. 1997. Salmonella typhi stimulation of human intestinal epithelial cells induces secretion of epithelial cell-derived interleukin-6. Infect. Immun. 65:395-404.

29. Harcourt, J.L., and Offermann, M.K. 2000. Interferon alpha synergistically enhances induction of interleukin- 6 by double stranded RNA in HeLa cells. Eur. J. Biochem. 267:1-11.

30. Gewirtz, A.T., et al. 2000. Salmonella typhimurium induces epithelial IL-8 expression via $\mathrm{Ca}(2+)$-mediated activation of the NF-kappaB path- way. J. Clin. Invest. 105:79-92.

31. Clancy, J.P., Ruiz, F.E., and Sorscher, E.J. 1999. Adenosine and its nucleotides activate wild-type and R117H CFTR through an A2B receptor-coupled pathway. Am. J. Physiol. 276:C361-C369.

32. Si-Tahar, M., Merlin, D., Sitaraman, S., and Madara, J.L. 2000. Constitutive and regulated secretion of secretory leukocyte proteinase inhibitor by human intestinal epithelial cells. Gastroenterology. 118:1061-1071.

33. Schmitz, H., et al. 1999. Tumor necrosis factor-alpha (TNFalpha) regulates the epithelial barrier in the human intestinal cell line HT-29/B6. J. Cell. Sci. 112:137-146.

34. Shaywitz, A.J., and Greenberg, M.E. 1999. CREB: a stimulus-induced transcription factor activated by a diverse array of extracellular signals. Annu. Rev. Biochem. 68:821-861.

35. Roesler, W.J. 2000. What is a cAMP response unit? Mol. Cell. Endocrinol. 162:1-7.

36. Zidek, Z. 1999. Adenosine: cyclic AMP pathways and cytokine expression. Eur. Cytokine Netw. 10:319-328.

37. Reinisch, W., et al. 1999. Clinical relevance of serum interleukin-6 in Crohn's disease: single point measurements, therapy monitoring, and prediction of clinical relapse. Am. J. Gastroenterol. 94:2156-2164.

38. Biffl, W.L., et al. 1996. Interleukin-6 stimulates neutrophil production of platelet-activating factor. J. Leukoc. Biol. 59:569-574.

39. Hardin, J., Kroeker, K., Chung, B., and Gall, D.G. 2000. Effect of proinflammatory interleukins on jejunal nutrient transport. Gut. 47:184-191. 